\title{
Lipopolysaccharide-stimulated, NF-kB-, miRNA-146a- and miRNA-155-mediated molecular-genetic communication between the human gastrointestinal tract microbiome and the brain
}

\author{
Peter N. Alexandrov' ${ }^{1}$, Yuhai Zhao ${ }^{2,3}$, Wenhong Li²,4, Walter J. Lukiw ${ }^{2,5,6}$ \\ ${ }^{1}$ Russian Academy of Medical Sciences, Moscow, Russian Federation, ${ }^{2}$ LSU Neuroscience Center, Louisiana State University Health \\ Sciences Center, New Orleans, LA, USA, ${ }^{3}$ Department of Anatomy and Cell Biology, Louisiana State University Health Sciences \\ Center, New Orleans, LA, USA, ${ }^{4}$ Department of Pharmacology, School of Pharmacy, Jiangxi University of TCM, Nanchang, Jiangxi, \\ China, ${ }^{5}$ Department of Ophthalmology, Louisiana State University Health Sciences Center, New Orleans, LA, USA, ${ }^{6}$ Department \\ of Neurology, Louisiana State University Health Sciences Center, New Orleans, LA, USA
}

\begin{abstract}
Through the use of RNA sequencing, microRNA (miRNA) and messenger RNA (MRNA) microfluidic array analysis, LED Northern, Western and ELISA analysis and multiple bioinformatics algorithms we have discovered a novel route for pathogenic communication between the human gastrointestinal (GI)-tract microbiome and the brain. The evidence suggests that this pathogenic gut-brain circuit involves: (i) lipopolysaccharide (LPS) from the GI-tract resident enterotoxigenic Gram-negative bacteria Bacteroides fragilis (BF-LPS); (ii) LPS transit across the GI-tract barrier into the systemic circulation; (iii) transport of a highly pro-inflammatory systemic BF-LPS across the blood-brain barrier (BBB) into the brain-parenchyma and neuronal-cytoplasm; (iv) activation and signaling via the pro-inflammatory NF-kB (p50/p65) transcription-factor complex; (v) NF-kB-coupling and significant up-regulation of the inducible pro-inflammatory microRNA-146a (miRNA-146a) and microRNA-155 (miRNA-155); each containing multiple $N F-k B$ DNA-binding and activation sites in their immediate promoters; and (vi) subsequent down-regulation of miRNA-146a-miRNA-155 regulated mRNA targets such as that encoding complement factor $\mathrm{H}$ (CFH), a soluble complement control glycoprotein and key repressor of the innate-immune response. Down-regulated CFH expression activates the complement-system, the major non-cellular component of the innate-immune system while propagating neuro-inflammation. Other Gl-tract microbes and their highly complex pro-inflammatory exudates may contribute to this pathogenic GI-tract-brain pathway. We speculate that it may be significant that the first Gram-negative anaerobic bacterial species intensively studied as a potential contributor to the onset of Alzheimer's disease (AD), that being the bacillus Bacteroides fragilis appears to utilize damaged or leaky physiological barriers and an activated NF-kB (p50-p65) - pro-inflammatory miRNA-146a-miRNA-155 signaling circuit to convey microbiome-derived pathogenic signals into the brain.
\end{abstract}

Key words: Alzheimer's disease, Bacteroides fragilis (B. fragilis), complement factor $\mathrm{H}$ (CFH), dysbiosis, microRNA-146a, microRNA-155, NF-kB (p50/p65). 


\section{Overview: human Gl-tract microbiome and Bacteroides fragilis}

The gastrointestinal (GI) tract of Homo sapiens contains a complex microbiome consisting primarily of bacteria, with archaea, fungi, microbial eukaryotes, protozoa, viruses, and other microorganisms making up the balance $[3-5,7,8,16,19,25,26,89,90]$. Together with human host cells this microbiome comprises the entire meta-organism whose host interactions and symbiotic associations are critical to human health and disease $[4,16,18,35,72,88-90]$. These diseases include lethal, progressive, age-related, inflammatory neurodegenerative disorders of the human central nervous system (CNS) such as Alzheimer's disease $(A D)[4,5,9,35,58,60,72,76,82]$. Of the 52 currently recognized bacterial divisions, humans have co-evolved with just 2 dominant phyla: Bacteroidetes, representing $\sim 20-30 \%$ of all human GI-tract resident bacteria, and Firmicutes (about 70-80\%), with Actinobacteria ( $3 \%)$, Proteobacteria ( 1\%) and Verrucomicrobia ( $0.1 \%)$ making up the remainder $[4,5,7,61,78]$. These four major bacterial phyla represent the 'bacterial-core' of the human GI-tract microbiome $[4,7,8,78,82]$. The vast majority of all Gl-tract microbiota consists of Gram-negative anaerobic bacteria, and Bacteroidetes species represent the most abundant Gram-negative anaerobes, outnumbering Escherichia coli in abundance by about 100 to $1[3-8,14,15,61-64]$. Certain strains of Bacteroidetes species such as Bacteroides fragilis (B. fragilis), as a normal commensal microbe of the human Gl-tract, are thought to be ordinarily beneficial to human health due to their multiple capabilities: (i) to biosynthesize useful metabolic co-factors and products such as polysaccharides, transport proteins, volatile fatty acids and other nutrients [9,14,47,62,74]; (ii) to cleave dietary fiber into digestible short-chain fatty acids (SCFAs) that include acetate, propionate, and butyrate $[9,38,63,74]$; (iii) to function in the maintenance, development and homeostasis of the host immune system $[14,47,62,74,79]$; (iv) to support immunomodulation and protection against pathogens including potentially pathogenic GI tract bacteria $[9,14,29,63,79]$; and (v) to support glucose homeostasis $[8,9,13,63,69,72]$. Conversely, when enterotoxigenic strains of $B$. fragilis or their array of secretory neurotoxins leak through normally protective biophysiological-mucosal barriers they can cause substantial inflammatory pathology sys- temically that can contribute to significant mortality and morbidity $[15,29,57,63,72,90]$. Dietary intake of fiber may have a determinant role in regulating the composition, organization and stoichiometry of the GI-tract microbiome; for example Bacteroidetes species proliferate in porcine models fed high-fat diets that are deprived of sufficient dietary fiber $[13,22,32,57,69,83,84]$.

The secreted LPS derived from the outer membrane of $B$. fragilis (BF-LPS) is a remarkably neurotoxic and pro-inflammatory lipid-sugar lipoglycan consisting of a hydrophobic domain known as a lipid A 'endotoxin', a nonrepeating "core" oligosaccharide, and a distal polysaccharide 'O-antigen' [12,77]. Interestingly, of several different AD-associated proinflammatory mediators - including the pro-inflammatory cytokines interleukin $1 \beta$ (IL-1 $\beta$ ) and tumor necrosis factor $\alpha$ (TNF- $\alpha$ ), the neurotoxic $A \beta 40$ and $A \beta 42$ peptides, the combination of $A \beta 42+I L-1 \beta$ together, the LPS isolated from $E$. coli (EC-LPS) or $B$. fragilis (BF-LPS) - that were tested for their ability to induce the pro-inflammatory transcription factor NF-kB (p50/p65 complex) in human neuronal-glial (HNG) cells in primary co-culture, BF-LPS was by far the most potent. For example, $25 \mathrm{nM}$ doses of BF-LPS administered to HNG cells elicited an $\sim 11$-fold more of a pro-inflammatory response than the same quantity of $\mathrm{A} \beta 42$ peptide alone and a remarkable $\sim 27$-fold more of an inflammatory response than the pro-inflammatory cytokine TNF- $\alpha$ alone $[12,46,47]$. In addition to lipooligosaccharide (LOS) and lipopolysaccharide (LPS) generation, $B$. fragilis endotoxins are a leading cause of anaerobic bacteremia and sepsis driving systemic inflammatory distress through their generation of the highly pro-inflammatory $\sim 20 \mathrm{kDa}$ heat-labile zinc-dependent metalloprotease $B$. fragilis toxin (BFT) fragilysin $[10,14]$. Exposure to BFT results in the infiltration of a variety of inflammatory cells and also in the destruction of the mucosal epithelial cell layer $[10,62,63]$. The ability of $B$. fragilis strains to secrete BFT classifies these microbes as: (i) those that do not secrete BFT as the nontoxigenic form of $B$. fragilis (NTBF); and (ii) those that do secrete BFT are classified as the enterotoxigenic form of $B$. fragilis (ETBF) [14,62]. Interestingly, the ETBF form can (i) disrupt epithelial cells of Gl tract barriers via cleavage of the synaptic adhesion zonula adherens protein E-cadherin [14,24,39,62,63,65]; and (ii) induce clinical pathology, including intestinal diarrhea, celiac disease and systemic inflammation 
$[10,25-28,46,47,54,62,65,80]$. Indeed, under pathophysiological conditions within the aging $A D$ brain it might be expected that a pro-inflammatory cocktail of BF-LPS, BFT and other B. fragilis-derived exotoxins, amyloids and small non-coding RNAs (sncRNAs) would assault normal brain homeostasis together to elicit an even greater pro-inflammatory response in driving the neurodegenerative disease process (Fig. 1). It should also be kept in mind that other Gl-tract microbiome-resident microbes including fungus, protozoa, viruses, and other commensal microorganisms may also contribute highly neurotoxic exudates that can be strongly detrimental to the homeostasis of aging CNS neurons.

\section{Systemic inflammation and the propagation of GI-tract microbiome- derived signals}

Gl-tract microbiome-derived neurotoxin entry into the systemic circulation may be a precursor event to the onset of inflammatory neurodegeneration in the CNS and establishing pathogenic pathways of communication between the systemic and central innate-immune systems $[23,29,31,66]$. It is currently not well understood if Gl-tract barrier-disrupting proteolytic endotoxins such as BFT are able to propagate pathogenic actions via the systemic circulation to further disrupt the blood-brain barrier and transfer LPS, BFT, and other endotoxins into the cerebrovascular circulation to the brain parenchyma, neural cells and synaptic circuitry of the CNS. However, it has recently been reported that BF-LPS, BFT and amyloid peptides progressively alter neural cytoarchitecture, synaptic adhesion, affecting both the function and integrity of synapses - a series of processes that play critical roles in the disruption of functional inter-neuronal signaling throughout neuronal networks in $A D[33,39,42,48,57,65,76]$.

\section{Up-regulated microRNAs (miRNAs) and down-regulation of essential signaling components in Alzheimer's disease by Gl-tract microbiome-derived LPS}

The structure, function and evolution of miRNAs including their expanding list of critical regulatory roles in CNS development and age-related human neurodegenerative diseases such as $A D$ have been extensively reviewed and will not be dealt with further here $[1,2,34,41,45,49,51,70,83,88]$. Because miRNAs are relatively unstable in brain and retinal tissues with half-lives on the order of $\sim 1-3$ hrs, only significantly up-regulated miRNAs have been studied in short post-mortem interval (PMI) tissues of PMIs of 2-3 hrs or less; down-regulated miRNAs may simply be a consequence of the highly oxidative and pro-inflammatory degradative environment of actively degenerating neural tissues $[11,59,67]$. It has become abundantly clear that the major mode of action of miRNAs is to function as negative regulators of messenger RNA (mRNA)-mediated gene expression by binding to complementary ribonucleotide sequences in the 3'-untranslated region (3'-UTR) of target mRNAs, thus causing the repression of translation and/or degradation of that target mRNA $[21,59,73]$. Briefly, a small family of NF-kB (p50/p65 complex) induced pro-inflammatory microRNAs (miRNAs), including miRNA-9, miRNA-34a, miRNA-125b, miRNA$146 \mathrm{a}$, and miRNA-155, ultimately bind with the 3'-UTR of several target messenger RNAs (mRNAs) and thereby decrease their expression. Down-regulated mRNAs include those encoding the SH3-proline-rich multi-domain-scaffolding protein of the postsynaptic density (SHANK3), the triggering receptor expressed in myeloid/microglial cells (TREM2) and complement factor-H (CFH); similar deficiencies in these 3 proteins are also observed in sporadic AD brain $[20,27,28,36,89,90]$. The critical importance of a down-regulated CFH mRNA and protein, and hence the observed deficits in $\mathrm{CFH}$ expression and abundance in $A D$ in this scheme due to up-regulated pro-inflammatory miRNA-146a and miRNA-155 is particularly significant. CFH down-regulation is further discussed below due to its critical role in the regulation of the innate-immune response and the onset and propagation of neuro-degeneration and inflammatory signaling [16,36,43,50,52,53,55] (Fig. 1).

\section{Complement factor $\mathrm{H}$ as an important LPS-NF-kB-miRNA-146a-miRNA-155 end target}

Complementfactor H (CFH; GC01P196621), encoded from the regulator of complement activation gene cluster at human chromosome 1q31.3, is a large, structurally versatile and critical $155 \mathrm{kDa}$ soluble glycoprotein moderately expressed in the human neocortex and retina, and highly expressed in the gall bladder and the liver where the mature plasma form of CFH passes into the systemic circulation $[36,50,52,53]$; 


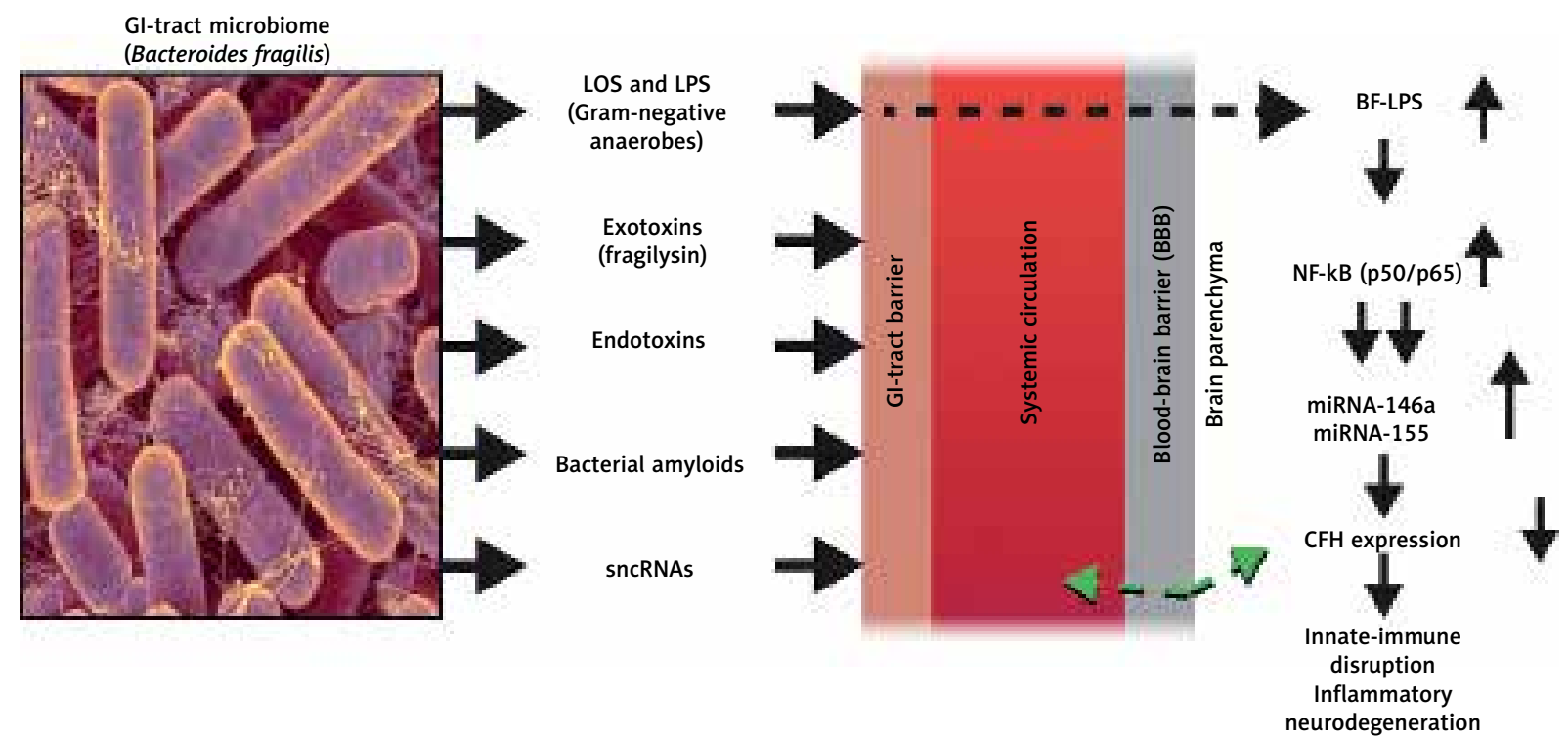

Fig. 1. Gram-negative anaerobes such as the abundant human gastrointestinal (GI)-tract resident Bacteroides fragilis (left panel; scanning electron microscopy (SEM) photograph of $B$. fragilis shown (9100x); original microphotograph courtesy of Rosa Rubicondior (http://rosarubicondior.blogspot.com/2014/11/evolving-cooperation-but-for-who-or-what.html; last accessed 4 July 2019), when stressed, release a broad spectrum of highly pro-inflammatory and potentially pathogenic molecules into the surrounding medium; these molecules comprise five major classes of secreted neurotoxins that include lipooligosaccharide (LOS) and lipopolysaccharide (LPS), exotoxins (such as fragilysin), endotoxins, bacterial amyloids, and small non-coding RNAs (sncRNAs) [7,12-14,24-27,87]. Normally, a highly dynamic Gl-tract barrier keeps bacteria and their neurotoxic exudates compartmentalized within the GI-tract; however, with aging and disease these barriers become 'leaky' and the same neurotoxins can easily transit GI-tract barriers to enter the systemic circulation. Gram-negative bacterial-derived LPS or other bacterial-derived neurotoxins in the systemic circulation give rise to a 'systemic inflammation' which may be a 'precursor event' to the development and/or establishment of Alzheimer's disease (AD) and other forms of progressive inflammatory neurodegeneration $[16,17,23,26,27,31,37,38,56,57,71,87]$. The extremely pro-inflammatory LPS of $B$. fragilis (BF-LPS) is exceptionally neurotoxic toward human CNS neurons in primary culture $[40,47]$. LPS in the systemic circulation can cross the blood-brain barrier (BBB) to access neural-susceptible brain compartments; there is also recent and compelling evidence that in the 5xFAD transgenic murine model of AD, LPS alone induces the opening of aging physiological barriers including the BBB $[6,54]$. Multiple laboratories have independently reported perivascular LPS localization in brain arteries and LPS accumulation within the brain parenchyma, within brain cell cytoplasm and surrounding neuronal cell nuclei with specific effects on neuron-specific gene expression [12,13,15,26,27,71,80,87-90]. BF-LPS rapidly and efficiently induces the pro-inflammatory NF-kB (p50/p65) complex in cultured human brain cells followed by increases in the abundance of the inducible pro-inflammatory miRNA-146a and miRNA-155 (both of these miRNAs containing active NF-kB-DNA binding sites in their immediate promoter [25-27,40,43,80,87-90]. One energetically favorable miRNA-146a and/or miRNA-155 mRNA target, both confirmed by bioinformatics and miRNA-mRNA-3'-UTR binding luciferase-reporter assay, is the 3'-untranslated region (3'-UTR) of complement factor $\mathrm{H}(\mathrm{CFH})$ mRNA resulting in, respectively, the down-regulation of CFH expression $[26,27,48,87]$. A down-regulated $\mathrm{CFH}$ is associated with the disruption of innate-immune signaling that supports inflammatory neurodegeneration and amyloidogenesis [17,34,36,45,55,75,89-91]. Although CFH is easily detected in the brain parenchyma and neuronal cytoplasm it is also highly abundant in the blood serum of the human systemic circulation, as may be some other pathogenic blood-cell-based biomarkers that may include specific kinase mutations and other polymorphisms [17,45,55,68,91; see also https://www.sigmaaldrich.com/catalog/product/sigma/c5813?lang=en\&region=US; ENSG000 00000971-CFH/ tissue; last accessed 4 July 2019]. Like for other biomarkers, CFH communication and/or exchange between the brain parenchyma and systemic circulation (green dashed arrow) is not well understood. Other up-regulated brain-enriched microRNAs and their down-regulated mRNA targets may also be involved in multiple pathogenic signaling mechanisms. 
https://www.proteinatlas.org/ENSG00000000971CFH/tissue; https://www.genecards.org/cgi-bin/card disp. pl? gene=CFH; last accessed 29 March 2019). Normal human plasma concentrations of CFH range between $\sim 200$ and $300 \mathrm{ug} / \mathrm{ml}$, the highest of any plasma complement protein, and as such represents the major human complement regulator in human blood, brain and retina [17,55; unpublished]. CFH is composed of $\sim 20$ tandem $\sim 60$ amino-acid 'complement control protein' modules each connected by 3-8 amino acid linkers whose principal function is to regulate the alternative pathway of complement system activation, a central component of the innate-immune system's natural defense against pathogens and microbial infection [27,28,36,52,88-90]. As the major immune regulator of the innate-immune system, CFH recognizes pathogen- and damage-associated molecular patterns (DAMPs) and initiates an immune response in coordination with innate and adaptive immunity [36,52,53,88-90]. When activated, the complement system unleashes powerful cytotoxic and pro-inflammatory mechanisms, and thus its stringent control is critical to allow the restoration of immune-homeostasis and the prevention of damage to host-tissues. Significant levels of CFH expression are maintained in the blood, brain and retinal tissues during development and aging, suggesting that this glycoprotein plays a role in protecting these compartments, cells and tissues from indiscriminate complement activation and innate-immune and/or inflammatory damage $[16,45,55,88-90]$. Brain $\mathrm{CFH}$, retinal $\mathrm{CFH}$ and plasma CFH levels have been found to be significantly decreased in mild cognitive impairment $(\mathrm{MCl})$, in late onset $A D(L O A D)$, in age-related macular degeneration (AMD) patients and in autoimmune disease when compared to age-matched controls $[17,27-29,45,55,89,90]$. The LPS-induced, NF-kB-stimulated pro-inflammatory microRNAs miRNA-146a and miRNA-155 regulate both brain and retinal CFH expression via very strong overlapping complementary RNA binding sites in the CFH 3' untranslated region (3'-UTR); interestingly the entire 232 ribonucleotide sequence of the human CFH mRNA 3'-UTR appears to contain multiple miRNA recognition features and highly complementary miRNA binding sites that may be alternately used in $\mathrm{CFH}$ regulation in the brain, retina and other neural or extra-neural tissues [17,27,28,89,90]. Together miRNA-146a and miRNA-155 recognize an overlapping miRNA regulatory control (MiRC) region in the CFH 3'-UTR
(5'-TTTAGTATTAA-3') to which either of these miRNAs may interact $[17,45]$. Progressive, pathogenic increases in specific miRNA binding to the entire 232 nucleotide CFH 3'-UTR appears to be a major regulator of $\mathrm{CFH}$ expression down-regulation, and the inflammatory pathology that characterizes both $A D$ and $A M D$. Besides miRNA-146a and miRNA-155, the involvement of other up-regulated miRNAs in controlling $\mathrm{CFH}$ expression is not known and cannot be excluded at this time $[17,45,55]$. Epigenetic-based gene therapies, including miRNA-based therapeutic strategies that target miRNA-146a and miRNA-155 using anti-miRNA-146a and/or anti-miRNA-155 strategies, and hence the regulation of CFH expression, may be greatly beneficial and advance the protective effects of CFH at inflammatory sites in CNS disease.

Importantly, CFH has several structurally related proteins also encoded at the regulator of complement activation gene cluster at human chromosome 1q31.3 that lack relevant complement regulatory activity - these are known as CFH-related (CFHR) proteins $[36,53,55]$. The balance between the actions of CFH and the CFHR proteins: (i) determines the degree of complement activation and the innate-immune response; and (ii) regulates the neurophysiological roles of CFH and CFHR in CNS health and disease $[36,75]$. The functional contributions of CFH and CFHR-mediated signaling to the innate-immune response in inflammatory neurodegeneration are currently not completely understood $[36,55,75]$.

\section{Concluding comments}

Recent experimental evidence continues to support the idea that the Gl-tract microbiome is capable of providing a rich source of potentially neurotoxic mediators capable of crossing the age-compromised or diseased Gl-tract endothelial cell barriers into the systemic circulation and then transit the BBB into the brain parenchyma. Age-related disease or dysfunction of these physiological barriers, poor diet and nutrition in the elderly, or the membrane- and adhesion protein-disruptive properties of the neurotoxins themselves may contribute to leakage of these pathogenic species into the systemic circulation and/or the establishment in the Gl-tract microbiome of bacterial dysbiosis that further supports the generation of these neurotoxic components. Gl-tract-derived toxins in the systemic circulation such as BF-LPS capable of crossing the BBB have strong potential to trigger the 
NF-kB (p50-p65)-miRNA-146a-miRNA-155 signaling system to convey Gl-tract microbiome-derived pathogenic signals into the brain. These have strong potential to down-regulate a select number critical mRNAs and their expression, such as for example, brain CFH, to induce disruption of the innate-immune response and inflammatory neurodegeneration (Fig. 1). Other up-regulated miRNAs appear to trigger neurological disease via the targeting and down-regulation of brain-enriched mRNAs, and hence gene expression, involved in the normal maintenance of the cytoskeleton and cytoarchitecture, both pre- and post-synaptic organization, neurotrophic support, amyloidogenesis and the clearance of AD-related lesions such as amyloid peptides $[28,34,59,67,83,88]$. We speculate that it may be significant that the first anaerobic bacterial species intensively studied as a potential contributor to the onset of $A D$, that being the Gram negative bacillus $B$. fragilis, appears to utilize damaged or leaky physiological barriers and an activated NF-kB (p50-p65)-proinflammatory miRNA-146a-miRNA-155 signaling system to convey microbiome-derived pathogenic signals into the brain $[28,34,57,63,85,86,89,90]$. Interestingly, the human neurotrophic virus herpes simplex 1 (HSV-1) also activates an NF-kB (p50-p65)-pro-inflammatory miRNA-146a signaling system during the initial infection of human brain neurons, but the nature and significance of this activation pathway, and whether or not this represents an immune evasion strategy by HSV-1 or the host cell, is currently not well understood $[24,30,44]$.

Lastly, the development of strategies for regulating and maintaining a healthy Gl-tract microbiome represents a valid, attainable and testable hypothesis for lowering an individual risk and the prevalence of progressive inflammatory neurodegeneration. Indeed, the modification of the Gl-tract microbiome composition and complexity via diet-based therapies, prebiotics or probiotics may support: (i) the establishment and maintenance of host-friendly, health-promoting and disease-reducing microbial composition; and (ii) provide new preventive and/or therapeutic options for a more effective clinical management of $A D$ and related forms of age-related inflammatory neurodegeneration $[4,5,23,38,40,61,81,89,90]$.

\section{Acknowledgements}

The analytical, experimental and statistical work in this short communication was presented in part at the Society for Neuroscience (SFN) Annual Meet- ing November 2018, San Diego CA, USA. Sincere thanks are extended to the late Drs. JM Hill $(J M H$; Louisiana State University), TPA Kruck (TPAK; University of Toronto), C. Bergeron (CB, University of Toronto) for helpful discussions on this research area and to F. Culicchia, C. Eicken, C. Hebel, B. Krishnan, K. Navel, and L. Wong for short postmortem interval (PMI) human and other mammalian brain tissues or extracts and to D. Guillot and A.I. Pogue for expert technical assistance. Thanks are also extended to the many neuropathologists, physicians and researchers of the US, Canada, Europe and Russia who have provided high quality, short postmortem interval (PMI) human CNS or extracted brain tissue fractions for scientific study. We would like to further thank the following 18 domestic and international brain banks, and their continuing cooperation, for access to high quality postmortem tissues and valuable analytical advice: the Autism Brain Net, Los Angeles, CA, USA; the Harvard University/McLean Hospital Tissue Center, Boston, MA, USA; Louisiana State University, New Orleans, LA, USA; the Lomonosov Institute, Moscow State University, Moscow, Russian Federation; the National Disease Research Interchange, Philadelphia, PA, USA; the National Institutes of Health NIH NeuroBioBank, comprised of tissues from the National Institute of Mental Health (NIMH), the Eunice Kennedy Shriver National Institute of Child Health and Human Development (NICHD), and the National Institute of Neurological Disorders and Stroke (NINDS), Bethesda, MD, USA; the Netherlands Brain Research Institute, Amsterdam, Netherlands; the New York State Institute for Basic Research, Staten Island, NY, USA; the Oregon Health Sciences University, Portland, OR, USA; the Southern Eye Bank, Metairie, LA, USA; the University of California, Irvine, CA, USA; the University of Kentucky Alzheimer's disease Brain Bank, Lexington, KY, USA; the University of Maryland Brain and Tissue Bank, Baltimore, MD, USA; the University of Massachusetts, Worcester, MA, USA; University of Pennsylvania School of Medicine, Philadelphia, PA, USA, and the University of Toronto Brain Bank, Toronto, ON, Canada. All authors agree on the content of this publication. Research on metal neurotoxicity, human and murine microRNAs, small noncoding RNA (sncRNA), proinflammatory and pathogenic signaling in the Lukiw laboratory involving the innate-immune response, neuroinflammation and amyloidogenesis in AD, ASD, PrD and in other human neurological disorders was supported 
through an unrestricted grant to the LSU Eye Center from Research to Prevent Blindness (RPB); the Louisiana Biotechnology Research Network (LBRN), the Alzheimer Association and NIH grants NEI EY006311, NIA AG18031 and NIA AG038834 (WJL).

\section{Ethics statement}

All acquisition, handling, experimental and analytical procedures involving postmortem human brain tissues were carried out in an ethical manner in strict accordance with the ethics review board policies at brain and tissue donor institutions and at the Louisiana State University (LSU) Health Sciences Center. Informed consent from next of kin was obtained at brain and tissue donor institutions for all tissue samples prior to autopsy and donation; coded postmortem brain tissue samples (containing no personal identifying information of the donors) were obtained from the 18 brain and tissue banks listed in the Acknowledgements section above. The ethical use of postmortem human brain tissues and their analyses were also carried out in strict accordance with the Institutional Biosafety Committee and the Institutional Review Board Committee (IBC/ IRBC) ethical guidelines IBC\#18059 and IRBC\#6774 at the LSU Health Sciences Center, New Orleans, LA 70112, USA.

\section{Disclosure}

The authors report no conflict of interest.

The experimental work in this paper was funded by the LSU Eye Center from Research to Prevent Blindness (RPB), the Louisiana Biotechnology Research Network (LBRN), the National Institutes of Health (NIH), Bethesda, MD, USA and the Alzheimer Association Chicago, IL, USA.

\section{References}

1. Alexandrov PN, Dua P, Lukiw WJ. Up-regulation of miRNA-146a in progressive, age-related inflammatory neurodegenerative disorders of the human CNS. Front Neurol 2014; 5: 181.

2. Alexandrov PN, Percy ME, Lukiw WJ. Chromosome 21-encoded microRNAs (mRNAs): impact on Down's syndrome and trisomy-21 linked disease. Cell Mol Neurobiol 2018; 38: 769-774.

3. Alkasir R, Li J, Li X, Jin M, Zhu B. Human gut microbiota: the links with dementia development. Protein Cell 2017; 8: 90-102.

4. Awany D, Allali I, Dalvie S, Hemmings S, Mwaikono KS, Thomford NE, Gomez A, Mulder N, Chimusa ER. Host and microbiome genome-wide association studies. Front Genet 2019; 9: 637.
5. Barko PC, McMichael MA, Swanson KS, Williams DA. The gastrointestinal microbiome: a review. J Vet Intern Med 2018; 32: 9-25.

6. Barton SM, Janve VA, McClure R, Anderson A, Matsubara JA, Gore JC, Pham W. LPS-induced opening of the blood brain barrier on aging 5xFAD mouse model. J Alzheimers Dis 2019; 67: 503-513.

7. Bhattacharjee S, Lukiw WJ. Alzheimer's disease and the microbiome. Front Cell Neurosci 2013; 7: 153.

8. Blum HE. The human microbiome. Adv Med Sci 2017; 62: 414420.

9. Chambers ES, Preston T, Frost G, Morrison DJ. Role of gut microbiota-generated short-chain fatty acids in metabolic and cardiovascular health. Curr Nutr Rep 2018; 7: 198-206.

10. Choi VM, Herrou J, Hecht AL, Teoh WP, Turner JR, Crosson S, Crosson S, Bubeck Wardenburg J. Activation of Bacteroides fragilis toxin by a novel bacterial protease. Nat Med 2016; 22: 563-567.

11. Clement C, Hill JM, Dua P, Culicchia F, Lukiw WJ. Analysis of RNA from Alzheimer's disease post-mortem brain tissues. Mol Neurobiol 2016; 53: 1322-1328.

12. Di Lorenzo F, De Castro C, Silipo A, Molinaro A. Lipopolysaccharide structures of Gram-negative populations in the gut-microbiota and effects on host interactions. FEMS Microbiol Rev 2019; 43: 257-272.

13. Durack J, Lynch SV. The gut microbiome: Relationships with disease and opportunities for therapy. J Exp Med 2019; 216: 20-40.

14. Fathi P, Wu S. Isolation, detection, and characterization of enterotoxigenic Bacteroides fragilis in clinical samples. Open Microbiol J 2016; 10: 57-63.

15. Foster JA, Lyte M, Meyer E, Cryan JF. Gut microbiota and brain function: an evolving field in neuroscience. Int J Neuropsychopharmacol 2016; 19.

16. Franceschi F, Ojetti V, Candelli M, Covino M, Cardone S, Potenza A, Simeoni B, Gabrielli M, Sabia L, Gasbarrini G, Lopetuso L, Scaldaferri F, Rossini PM, Gasbarrini A. Microbes and Alzheimer' disease: lessons from $\mathrm{H}$. pylori and GUT microbiota. Eur Rev Med Pharmacol Sci 2019; 23: 426-430.

17. Gezen-Ak D, Dursun E, Hanağası H, Bilgiç B, Lohman E, Araz ÖS, Atasoy IL, Alaylıoğlu M, Önal B, Gürvit H, Yılmazer S. BDNF, TNF $\alpha$, HSP90, CFH, and IL-10 serum levels in patients with early or late onset Alzheimer's disease or mild cognitive impairment. J Alzheimers Dis 2013; 37: 185-195.

18. Ghaisas S, Maher J, Kanthasamy A. Gut microbiome in health and disease: Linking the microbiome-gut-brain axis and environmental factors in the pathogenesis of systemic and neurodegenerative diseases. Pharmacol Ther 2016; 158: 52-62.

19. Giau VV, Wu SY, Jamerlan A, An SSA, Kim SY, Hulme J. Gut microbiota and their neuroinflammatory implications in Alzheimer's disease. Nutrients 2018; 10. pii: E1765.

20. Guilmatre A, Huguet G, Delorme R, Bourgeron T. The emerging role of SHANK genes in neuropsychiatric disorders. Dev Neurobiol 2014; 74: 113-122.

21. Guo H, Ingolia NT, Weissman JS, Bartel DP. Mammalian microRNAs predominantly act to decrease target mRNA levels. Nature 2010; 466: 835-840.

22. Heinritz SN, Weiss E, Eklund M, Aumiller T, Heyer CM, Messner S, Rings A, Louis S, Bischoff SC, Mosenthin R. Impact of a high- 
fat or high-fiber diet on intestinal microbiota and metabolic markers in a pig model. Nutrients 2016; 8: E317.

23. Heneka MT, Carson MJ, El Khoury J, Landreth GE, Brosseron F, Feinstein DL, Jacobs AH, Wyss-Coray T, Vitorica J, Ransohoff RM, Herrup K, Frautschy SA, Finsen B, Brown GC, Verkhratsky A Yamanaka K, Koistinaho J, Latz E, Halle A, Petzold GC, Town T, Morgan D, Shinohara ML, Perry VH, Holmes C, Bazan NG, Brooks DJ, Hunot S, Joseph B, Deigendesch N, Garaschuk O, Boddeke E, Dinarello CA, Breitner JC, Cole GM, Golenbock DT, Kummer MP. Neuroinflammation in Alzheimer's disease. Lancet Neurol 2015; 14: 388-405.

24. Hill JM, Zhao Y, Clement C, Neumann DM, Lukiw WJ. HSV-1 infection of human brain cells induces miRNA-146a and Alzheimer-type inflammatory signaling. Neuroreport 2009; 20: 1500 1505

25. Hill JM, Clement C, Pogue Al, Bhattacharjee S, Zhao Y, Lukiw WJ. Pathogenic microbes, the microbiome, and Alzheimer's disease (AD). Front Aging Neurosci 2014; 6: 127.

26. Hill JM, Bhattacharjee S, Pogue Al, Lukiw WJ. The gastrointestinal tract microbiome and potential link to Alzheimer's disease. Front Neurol 2014; 5: 43.

27. Hill JM, Lukiw WJ. Microbial-generated amyloids and Alzhei mer's disease (AD). Front Aging Neurosci 2015; 7: 9.

28. Hill JM, Pogue Al, Lukiw WJ. Pathogenic microRNAs common to brain and retinal degeneration; recent observations in Alzheimer's disease and age-related macular degeneration. Front Neurol 2015; 6: 232.

29. Hofer U. Microbiome: B. fragilis and the brain. Nat Rev Microbiol 2014; 12: 76-77.

30. Hogestyn JM, Mock DJ, Mayer-Proschel M. Contributions of neurotropic human herpesviruses herpes simplex virus 1 and human herpesvirus 6 to neurodegenerative disease pathology. Neural Regen Res 2018; 13: 211-221.

31. Holmes C. Review: systemic inflammation and Alzheimer's disease. Neuropathol Appl Neurobiol 2013; 39: 51-68.

32. Holscher HD. Dietary fiber and prebiotics and the gastrointestinal microbiota. Gut Microbes 2017; 8: 172-184.

33. Ilic K, Mlinac-Jerkovic K, Jovanov-Milosevic N, Simic G, Habek N, Bogdanovic N, Kalanj-Bognar S. Hippocampal expression of cell-adhesion glycoprotein neuroplastin is altered in Alzheimer's disease. J Cell Mol Med 2019; 23: 1602-1607.

34. Jaber V, Zhao Y, Lukiw WJ. Alterations in micro RNA-messenger RNA (miRNA-mRNA) coupled signaling networks in sporadic Alzheimer's disease (AD) hippocampal CA1. J Alzheimers Dis Parkinsonism 2017; 7: pii: 312.

35. Jiang C, Li G, Huang P, Liu Z, Zhao B. The gut microbiota and Alzheimer's disease. J Alzheimers Dis 2017; 58: 1-15.

36. Józsi M, Schneider AE, Kárpáti É, Sándor N. Complement factor $\mathrm{H}$ family proteins in their non-canonical role as modulators of cellular functions. Semin Cell Dev Biol 2019; 85: 122-131.

37. Keaney J, Campbell M. The dynamic blood-brain barrier. FEBS 2015: 282: 4067-4079.

38. Kowalski K, Mulak A. Brain-gut-microbiota axis in Alzheimer's disease. J Neurogastroenterol Motil 2019; 25: 48-60.

39. Leshchyns'ka I, Sytnyk V. Synaptic cell adhesion molecules in Alzheimer's disease. Neural Plast 2016; 64: 275237.
40. Li YY, Cui JG, Hill JM, Bhattacharjee S, Zhao Y, Lukiw WJ. Increased expression of miRNA-146a in Alzheimer's disease transgenic mouse models. Neurosci Lett 2011; 487: 94-98.

41. Lim LP, Glasner ME, Yekta S, Burge CB, Bartel DP. Vertebrate microRNA genes. Science 2003; 299: 1540.

42. Lin CS, Chang CJ, Lu CC, Martel J, Ojcius DM, Ko YF, Young JD, Lai HC. Impact of the gut microbiota, prebiotics, and probiotics on human health and disease. Biomed J 2014; 37: 259-268.

43. Lukiw WJ, Zhao Y, Cui JG. An NF-kB-sensitive miRNA-146amediated inflammatory circuit in Alzheimer's disease and in stressed human brain cells. J Biol Chem 2008; 283: 31315-31322.

44. Lukiw WJ, Cui JG, Yuan LY, Bhattacharjee PS, Corkern M, Clement C, Kammerman EM, Ball MJ, Zhao Y, Sullivan PM, Hill JM. Acyclovir or A $\beta 42$ peptides attenuate HSV-1-induced miRNA-146a levels in human primary brain cells. Neuroreport 2010; 21: $922-927$

45. Lukiw WJ, Alexandrov PN. Regulation of complement factor $\mathrm{H}$ (CFH) by multiple miRNAs in Alzheimer's disease (AD) brain. Mol Neurobiol 2012; 46: 11-19.

46. Lukiw WJ. The microbiome, microbial-generated pro-inflammatory neurotoxins, and Alzheimer's disease. J Sport Health Sci 2016; 5: 393-396.

47. Lukiw WJ. Bacteroides fragilis lipopolysaccharide (BF-LPS) and inflammatory signaling in Alzheimer's disease. Front Microbiol 2016; 7: 1544

48. Lukiw WJ, Cong L, Jaber V, Zhao Y. Microbiome-derived lipopolysaccharide (LPS) selectively inhibits neurofilament light chain (NF-L) gene expression in human neuronal-glial (HNG) cells in primary culture. Front Neurosci 2018; 12: 896.

49. Maiese K. MicroRNAs for the treatment of dementia and Alzheimer's disease. Curr Neurovasc Res 2019; 16: 1-2.

50. Mandal MN, Ayyagari R. Complement factor H: spatial and temporal expression and localization in the eye. Invest Ophthalmol Vis Sci 2006; 47: 4091-4097.

51. Maoz R, Garfinkel BP, Soreq H. Alzheimer's disease and ncRNAs. Adv Exp Med Biol 2017; 978: 337-361.

52. Marszałek M. Alzheimer's disease against peptides products of enzymatic cleavage APP protein: Biological, pathobiological and physico-chemical properties of fibrillating peptides. Postepy Hig Med Dosw 2017; 71: 398-410

53. Medjeral-Thomas N, Pickering MC. The complement factor H-related proteins. Immunol Rev 2016; 274: 191-201.

54. Montagne A, Zhao Z, Zlokovic BV. Alzheimer's disease: a matter of blood-brain barrier dysfunction? J Exp Med 2017; 214: 3151-3169.

55. Pouw RB, Brouwer MC, Geissler J, van Herpen LV, Zeerleder SS, Wuillemin WA, Wouters D, Kuijpers TW. Complement factor $\mathrm{H}$-related protein 3 serum levels are low compared to factor $\mathrm{H}$ and mainly determined by gene copy number variation in CFHR3. PLoS One 2016; 11: e0152164.

56. Rajendran L, Paolicelli RC. Microglia-mediated synapse loss in Alzheimer's disease. J Neurosci 2018; 38: 2911-2919.

57. Rakic S, Hung YMA, Smith M, So D, Tayler HM, Varney W, Wild J, Harris S, Holmes C, Love S, Stewart W, Nicoll JAR, Boche D. Systemic infection modifies the neuro-inflammatory response in late stage Alzheimer's disease. Acta Neuropathol Commun 2018; 6: 88 
58. Rinninella E, Raoul P, Cintoni M, Franceschi F, Miggiano GAD, Gasbarrini A, Mele MC. What is the healthy gut microbiota composition? a changing ecosystem across age, environment, diet, and diseases. Microorganisms 2019; 7: pii: E14.

59. Rüegger S, Großhans H. MicroRNA turnover: when, how, and why. Trends Biochem Sci. 2012; 37: 436-446.

60. Sampson TR, Mazmanian SK. Control of brain development, function, and behavior by the microbiome cell host microbe. Cell Host Microbe 2016; 17: 565-576.

61. Sarkar RS, Banerjee S. Gut microbiota in neurodegenerative disorders. J Neuroimmunol 2019; 328: 98-104.

62. Sears CL. Enterotoxigenic Bacteroides fragilis: a rogue among symbiotes. Clin Microbiol Rev 2009; 22: 349-369.

63. Sears CL, Geis AL, Housseau F. Bacteroides fragilis subverts mucosal biology: from symbiont to colon carcinogenesis. J Clin Invest 2014; 124: 4166-4172.

64. Seksik P, Landman C. Understanding microbiome data. Dig Dis 2015; 33: 11-16.

65. Seong E, Yuan L, Arikkath J. Cadherins and catenins in dendrite and synapse morphogenesis. Cell Adh Migr 2015; 9: 202-213.

66. Serpente M, Bonsi R, Scarpini E, Galimberti D. Innate immune system and inflammation in Alzheimer's disease: from pathogenesis to treatment. Neuroimmunomodulation 2014; 21: 79-87.

67. Sethi P, Lukiw WJ. Micro-RNA abundance and stability in human brain: specific alterations in Alzheimer's disease temporal lobe neocortex. Neurosci Lett 2009; 459: 100-104.

68. Silva AS, Teixeira AG, Bavia L, Lin F, Velletri R, Belfort R Jr, Isaac L Plasma levels of complement proteins from the alternative pathway in patients with age-related macular degeneration are independent of Complement Factor H Tyr402 His polymorphism. Mol Vis 2012; 18: 2288-2299.

69. Simpson HL, Campbell BJ. Review article: dietary fiber-microbiota interactions. Aliment Pharmacol Ther 2015; 42: 158-179.

70. Swarbrick S, Wragg N, Ghosh S, Stolzing A. Systematic review of miRNA as biomarkers in Alzheimer's disease. Mol Neurobio 2019.

71. Sweeney MD, Sagare AP, Zlokovic BV. Blood-brain barrier breakdown in Alzheimer disease and other neurodegenerative disorders. Nat Rev Neurol 2018; 14: 133-150.

72. Ticinesi A, Tana C, Nouvenne A. The intestinal microbiome and its relevance for functionality in older persons. Curr Opin Clin Nutr Metab Care 2019; 22: 4-12.

73. Towler BP, Jones CI, Newbury SF. Mechanisms of regulation of mature miRNAs. Biochem Soc Trans 2015; 43: 1208-1214.

74. Troy EB, Kasper DL. Beneficial effects of Bacteroides fragilis polysaccharides on the immune system. Front Biosci (Landmark Ed) 2010; 15: 25-34.

75. Van Dyken P, Lacoste B. Impact of metabolic syndrome on neuroinflammation and the blood-brain barrier. Front Neurosci 2018; 12: 930.

76. Wang J, Song Y, Chen Z, Leng SX. Connection between systemic inflammation and neuroinflammation underlies neuroprotective mechanism of several phytochemicals in neurodegenerative diseases. Oxid Med Cell Longev 2018; 2018: 1972714.

77. Whitfield C, Trent MS. Biosynthesis and export of bacterial lipopolysaccharides. Annu Rev Biochem 2014; 83: 99-128.
78. Youssef NH, Couger MB, McCully AL, Criado AE, Elshahed MS. Assessing the global phylum level diversity within the bacterial domain: a review. J Adv Res 2015; 6: 269-282.

79. Zafar H, Saier MH Jr. Comparative genomics of transport proteins in seven Bacteroides species. PLoS One 2018; 13: e0208151.

80. Zhan X, Stamova B, Jin LW, DeCarli C, Phinney B, Sharp FR. Gram-negative bacterial molecules associate with Alzheimer disease pathology. Neurology 2016; 87: 2324-2332.

81. Zhan X, Stamova B, Sharp FR. Lipopolysaccharide associates with amyloid plaques, neurons and oligodendrocytes in Alzheimer's disease brain. Front Aging Neurosci 2018; 10: 42.

82. Zhao Y, Lukiw WJ. Microbiome-generated amyloid and potential impact on amyloidogenesis in Alzheimer's disease (AD). J Nat Sci 2015; 1: pii: e138.

83. Zhao Y, Pogue Al, Lukiw WJ. MicroRNA (miRNA) signaling in the human CNS in sporadic Alzheimer's disease (AD) - novel and unique pathological features. Int J Mol Sci 2015; 16: 3010530116.

84. Zhao Y, Dua P, Lukiw WJ. Microbial sources of amyloid and relevance to amyloidogenesis and Alzheimer's disease (AD). J Alzheimers Dis Parkinsonism 2015; 5: 177.

85. Zhao Y, Cong L, Jaber V, Lukiw WJ. Microbiome-derived lipopolysaccharide enriched in the perinuclear region of Alzheimer's disease brain. Front Immunol 2017; 8: 1064.

86. Zhao Y, Cong L, Lukiw WJ. LPS accumulates in neocortical neurons of $A D$ brain and impairs transcription in human neuronal-glial primary co-cultures. Front Aging Neurosci 2017; 9: 407.

87. Zhao Y, Jaber V, Lukiw WJ. Secretory products of the human GI tract and their potential impact on Alzheimer's disease (AD): detection of lipopolysaccharide (LPS) in AD hippocampus. Front Cell Infect Microbiol 2017; 7: 318.

88. Zhao Y, Cong L, Lukiw WJ. Plant and animal microRNAs (miRNAs) and their potential for inter-kingdom communication. Cell Mol Neurobiol 2018; 38: 133-140.

89. Zhao Y, Lukiw WJ. Bacteroidetes neurotoxins and inflammatory neurodegeneration. Mol Neurobiol 2018; 55: 9100-9107.

90. Zhao Y, Lukiw WJ. Microbiome-mediated upregulation of microRNA-146a in sporadic Alzheimer's disease. Front Neurol 2018; 9: 145.

91. Pluta R, Ułamek-Kozioł M, Januszewski S, Czuczwar SJ. Platelets, lymphocytes and erythrocytes from AD patients: the quest for blood cell-based biomarkers. Folia Neuropathol 2018; 56: $14-20$. 